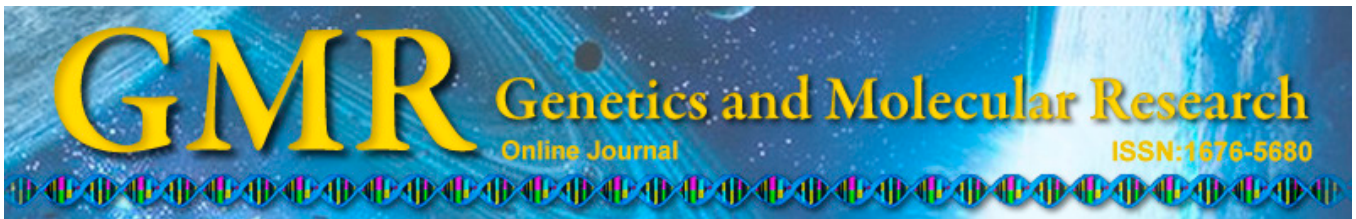

\title{
Intracranial aneurysm risk factor genes: relationship with intracranial aneurysm risk in a Chinese Han population
}

\author{
L.T. Zhang ${ }^{1 *}$, F.J. Wei ${ }^{2 *}$, Y. Zhao ${ }^{1}$, Z. Zhang ${ }^{1}$, W.T. Dong ${ }^{1}$, Z.N. Jin ${ }^{1}$, \\ F. Gao ${ }^{1}$, N.N. Gao ${ }^{1}$, X.W. Cai ${ }^{1}$, N.X. Li ${ }^{1}$, W. Wei ${ }^{1}$, F.S. Xiao ${ }^{1}$, S.Y. Yue ${ }^{1}$, \\ J.N. Zhang ${ }^{1}$, S.Y. Yang', W.D. Li ${ }^{2}$ and X.Y. Yang ${ }^{1}$ \\ ${ }^{1}$ Department of Neurosurgery, Tianjin Medical University General Hospital, \\ Tianjin, China \\ ${ }^{2}$ Research Center of Basic Medical Sciences, Tianjin Medical University, \\ Tianjin, China \\ *These authors contributed equally to this study. \\ Corresponding authors: X.Y. Yang / W.D. Li \\ E-mail: yangxinyu@tijmu.edu.cn / liweidong98@hotmail.com6
}

Genet. Mol. Res. 14 (2): 6865-6878 (2015)

Received September 30, 2014

Accepted February 27, 2015

Published June 18, 2015

DOI http://dx.doi.org/10.4238/2015.June.18.30

\begin{abstract}
Few studies have examined the genes related to risk factors that may contribute to intracranial aneurysms (IAs). This study in Chinese patients aimed to explore the relationship between IA and 28 gene loci, proven to be associated with risk factors for IA. We recruited 119 patients with aneurysms and 257 controls. Single factor and logistic regression models were used to analyze the association of IA and IA rupture with risk factors. Twenty-eight single nucleotide polymorphisms (SNPs) in 22 genes were genotyped for the patient and control groups. SNP genotypes and allele frequencies were analyzed by the chi-square test. Logistic regression analysis identified hypertension as a factor that increased IA risk ( $\left.\mathrm{P}=1.0 \times 10^{-4} ; \mathrm{OR}, 2.500 ; 95 \% \mathrm{CI}, 1.573-3.972\right)$; IA was associated with two SNPs in the TSLC2A9 gene: rs 7660895
\end{abstract}


$(\mathrm{P}=0.007 ;$ OR, 1.541; 95\%CI, 1.126-2.110); and in the TOX gene: rs11777927 (P = 0.013; OR, 1.511; 95\%CI, 1.088-2.098). Subsequent removal of the influence of family relationship identified between 12 of 119 patients enhanced the significant association of these SNPs with IA $(\mathrm{P}=0.001$; OR, 1.691; 95\%CI, 1.226-2.332; and $\mathrm{P}=0.006$; OR, 1.587; $95 \% \mathrm{CI}, 1.137-2.213$ for rs 7660895 and rs 11777927 , respectively). Furthermore, the minor allele of rs7660895 (A) was also associated with IA rupture ( $\mathrm{P}=0.007$; OR, 2.196; 95\%CI, 1.230-3.921). Therefore, hypertension is an independent risk factor for IA. Importantly, the TSLC2A9 (rs7660895) and TOX (rs11777927) gene polymorphisms may be associated with formation of IAs, and rs 7660895 may be associated with IA rupture.

Key words: Intracranial aneurysm; Gene; SNP; Risk factor

\section{INTRODUCTION}

Cerebral aneurysms are common lesions with a prevalence of 1 to $5 \%$ in adults and an annual rupture rate of 0 to $1 \%$ (Vlak et al., 2011). Ruptured aneurysms, the most common cause of non-traumatic subarachnoid hemorrhage, can be catastrophic and have high rates of mortality and morbidity. The prognosis following a ruptured intracranial aneurysm (IA) is very poor: about $30 \%$ of patients with subarachnoid hemorrhage (SAH) die before reaching the hospital; $50 \%$ of patients die within 1 month; and among those who survive, nearly $50 \%$ experience permanent neurological deficits (Katati et al., 2007). Despite the high incidence of IAs and the disastrous outcomes of their rupture, relatively little is known about the molecular pathogenesis of this disorder. IA formation is considered a complex disease process that is influenced by both genetic and environmental risk factors, including age, (female) gender, excessive drinking, smoking, and hypertension (Feigin et al., 2005). The frequency of IAs increases with age beyond the third decade, and they are 1.6 times more common in women (Wardlaw and White, 2000). At least 10\% of patients have a family history of IAs, which are associated with certain genetic conditions (Ruigrok et al., 2005); several Mendelian disorders, such as autosomal-dominant polycystic kidney disease and Ehlers-Danlos syndrome type IV, are associated with an increased risk of IA formation. These observations together support a role for genetic factors in the pathogenesis of IA.

Over the last decade, application of the hypothesis "common variants, common disease" has greatly aided in the identification of common variants associated with polygenic diseases. Through candidate gene approaches and genome-wide association studies (GWAS) several common variants have been identified as being associated with an increased risk of developing IAs; however, few of these associations were consistently replicated. Non-replication might occur because of poor statistical power or differences in allele frequencies across different populations. On the other hand, genes have been reliably implicated as being involved in the biology of well-known risk factors for IA [e.g., arterial hypertension, hyperlipidemia, type II diabetes mellitus (T2DM), obesity, high serum urate, and inflammation]. To the best of our knowledge, however, studies evaluating the role of risk factor candidate genes in the formation of IA are lacking to date.

The number of reports examining susceptibility loci for IA among Asian populations 
is particularly limited; therefore, we conducted a case-control association study using 119 patients with IA and 257 controls, and screened a total of 28 single-nucleotide polymorphisms (SNPs) in the genes CAMTA1, ABI2, PCAF, SLC2A9, ABCG2, SLC17A1, PKHD1, ESR1, NUB1, SLC30A8, CDKN2A/B, SIRT1, TCF7L2, IGF1, TBX5, SLITRK5, TOX, FTO, KCNJ15, $S F I 1, M Y H 9$, and $P D G F B$, which have been considered potential genetic risk factors of hypertension, obesity, T2DM, gout, and inflammation.

\section{MATERIAL AND METHODS}

\section{Study population}

The study population consisted of 119 patients with IA who were diagnosed by digital subtraction angiography, computerized tomography angiogram (CTA), as confirmed by both a neurosurgeon and a radiologist, or by neurosurgical operations in the Tianjin Medical University General Hospital. The control group included 257 volunteers who met the following criteria: 1) lack of IA, which was confirmed by CTA review by both a neurosurgeon and a radiologist (subjects with suspected positive results were excluded from the study);2) no medical history of any stroke including IA or SAH; and 3) no family history of IA or SAH among first-degree relatives. All patients and control subjects were residents of Tianjin City and members of the Han ethnic group. Patients and controls were matched for area of residence to eliminate the effect of population stratification by heterogeneity. The demographic and clinical parameters of both groups are summarized in Table 1 . The study was approved by the Ethics Committee of Tianjin Medical University. All subjects were interviewed for their risk factor profile, including past medical history, family history, smoking habit, and alcohol consumption. Smoking habit was defined as current smokers of $\geq 1$ cigarette per day; former smokers; or nonsmokers. For statistical analysis, current smokers and former smokers were grouped together as smokers. Drinkers were defined as regular drinkers who drank $150 \mathrm{~mL}$ of alcohol per week.

Table 1. Demographic and clinical parameters of case subjects and controls.

\begin{tabular}{|c|c|c|c|c|c|}
\hline & $\begin{array}{l}\text { Patients with IA } \\
\quad(\mathrm{N}=119)\end{array}$ & $\begin{array}{l}\text { Control IA } \\
(\mathrm{N}=257)\end{array}$ & $\begin{array}{l}\text { Univariate } \\
\text { analysis }(\mathrm{P})\end{array}$ & $\begin{array}{l}\text { Multivariate } \\
\text { analysis }(\mathrm{P})\end{array}$ & $\begin{array}{c}\text { Multivariate } \\
\text { analysis }[\mathrm{OR}(95 \% \mathrm{CI})]\end{array}$ \\
\hline Age (years) & $53.34 \pm 11.420$ & $51.17 \pm 10.519$ & 0.072 & - & - \\
\hline Female & $70(58.8)$ & $117(44.5)$ & 0.016 & 0.121 & $1.444(0.907-2.298)$ \\
\hline Hypertension & $76(63.9)$ & $99(38.5)$ & $4.6 \times 10^{-6}$ & $1.0 \times 10^{-4}$ & $2.500(1.573-3.972)$ \\
\hline Hypercholesterolemia & $24(20.2)$ & $45(17.5)$ & 0.536 & - & - \\
\hline Diabetes & $17(14.3)$ & $19(7.4)$ & 0.035 & 0.119 & $1.787(0.861-3.711)$ \\
\hline Smoking & $41(34.5)$ & $116(45.1)$ & 0.051 & - & - \\
\hline Alcohol & $30(25.2)$ & $77(30.0)$ & 0.342 & - & - \\
\hline Heart disease & $13(10.9)$ & $11(4.3)$ & 0.014 & 0.085 & $2.155(0.900-5.164)$ \\
\hline Family relationships & $12(10.1)$ & - & - & - & - \\
\hline
\end{tabular}

*Data are reported as $\mathrm{N}(\%)$ of patients or means $\pm \mathrm{SD}$. P values show differences between cases and control individuals.

\section{SNP selection}

We selected candidate SNPs associated with epidemiologic risk factors for IA as those having a $\mathrm{P}<5 \times 10^{-8}$ from published GWAS. Our primary analyses examined 28 SNPs as- 
sociated with well-established IA risk factors, including hypertension, hypercholesterolemia, obesity, T2DM, gout, polycystic kidney disease, and inflammation, and those SNPs previously found to be associated with IA in GWAS.

\section{DNA extraction and SNP genotyping}

Blood ( $\sim 2 \mathrm{~mL})$ was collected in EDTA collection tubes from each subject and stored at $-20^{\circ} \mathrm{C}$ until use. Genomic DNA was extracted from the stored blood using a DNA extraction kit (Bio Teke Corporation, Beijing, China). SNP genotyping was performed using the Sequenom MassARRAY platform according to manufacturer instructions (Sequenom, San Diego, CA, USA). PCR conditions for initial target amplification were as follows: $94^{\circ} \mathrm{C}$ for $15 \mathrm{~min}$; and 45 cycles of $94^{\circ} \mathrm{C}$ for $20 \mathrm{~s}, 56^{\circ} \mathrm{C}$ for $30 \mathrm{~s}$, and $72^{\circ} \mathrm{C}$ for $1 \mathrm{~min}$. The PCR products were analyzed by $1.0 \%$ agarose gel electrophoresis prior to genotyping.

\section{Quality control (QC)}

The sample QC threshold was set at 50\% (five of nine successful genotype calls). The SNP QC threshold was set at $80 \%$. All SNPs were tested for Hardy-Weinberg equilibrium at the 5\% significance level in the female cohort. SNPs that were not in HWE in the female control cohort were excluded from all analyses. In the IA group, DNA extraction and SNP genotyping passed QC measures for 116 subjects, of whom 82 subjects had ruptured IAs, while in the control group, informative results were obtained from 251 subjects.

\section{Statistical analysis}

All statistical analyses were carried out using the SPSS statistical software, version 17.0 (SPSS Inc., Chicago, IL, USA) for Windows. Differences between continuous variables and between categorical variables were assessed by the Student $t$-test and Pearson $\chi^{2}$ test, respectively. Univariate analyses were performed to calculate crude P values. Subsequently, we performed a multivariate backward stepwise logistic regression analysis to identify independent risk factors. The Pearson $\chi^{2}$ test was also used to examine any differences in allelic and genotypic frequencies between different groups, and odds ratios (ORs) and 95\% confidence intervals (CIs) were calculated. Two-sided $\mathrm{P}$ values were used, with $\mathrm{P}<0.05$ considered to be statistically significant. The Fisher exact $\mathrm{P}$ value was used when appropriate.

\section{RESULTS}

\section{Demographic and clinical parameters of participants}

The study population consisted of 119 patients with IA and 257 matched control subjects. Demographic characteristics of patients with IA and controls are summarized in Table 1. There were no statistically significant differences between groups with respect to hypercholesterolemia, diabetes, smoking, alcohol consumption, or heart disease. Family relationships were identified among 12 of 119 patients. Logistic regression analysis identified hypertension as a factor that increased the risk for IA ( $\mathrm{P}=1.0 \times 10^{-4}$; OR, 2.500; 95\%CI, 1.573-3.972). Therefore, hypertension is an independent risk factor for IAs. 


\section{Characteristics of aneurysms}

Clinical characteristics of aneurysms, including location sites and shapes, are shown in Table 2. A total of 138 aneurysms were detected in 119 patients. Of these, 16 patients $(13.4 \%)$ had several aneurysms: 13 had two each $(10.9 \%)$, three had three aneurysms each (2.5\%), and 102 had one. Overall, 67 aneurysms (48.6\%) were located in the internal carotid artery; $34(24.6 \%)$ were located in the anterior cerebral and anterior communicating arteries; $17(12.3 \%)$ were located in the middle cerebral artery; six (4.3\%) in the posterior communicating artery; five (3.6\%) in the posterior cerebral artery; four (2.9\%) in the basilar artery; and five $(3.6 \%)$ were located in the vertebral artery. Intracranial saccular aneurysms were found in $122(88.4 \%)$ of the 138 aneurysms.

Univariate analysis determined that age, gender, hypertension, cigarette smoking, hypercholesterolemia, T2DM, and daily drinking were not associated with an increased risk of aneurysm rupture (Table 3).

\section{Table 2. Clinical characteristics of aneurysms.}

\begin{tabular}{lc}
\hline Characteristic & No. IA $(\mathrm{N}=138)$ \\
\hline Site of aneurysm & \\
ICA & $67(48.6)$ \\
MCA & $17(12.3)$ \\
ACA/ACoA & $34(24.6)$ \\
PCoA & $6(4.3)$ \\
PCA & $5(3.6)$ \\
BA & $4(2.9)$ \\
VA & $5(3.6)$ \\
Shape of aneurysm & $122(88.4)$ \\
Sacular & $11(8.0)$ \\
Fusiform & $5(3.6)$ \\
Others & $103(74.6)$ \\
IA type & $35(25.4)$ \\
Single aneurysm & $26(18.8)$ \\
Multiple aneurysms & $9(6.5)$ \\
Two positions & \\
Three positions &
\end{tabular}

Data are reported as N (\%) of No. IA. ICA: internal carotid artery, ACA: anterior cerebral artery, ACoA: anterior communicating artery, MCA: middle cerebral artery, PCoA: posterior communicating artery, PCA: posterior cerebral artery, BA: basilar artery, VA: vertebral artery.

Table 3. Risk factors for rupture of an intracranial aneurysm.

\begin{tabular}{|c|c|c|c|c|c|}
\hline & $\begin{array}{l}\text { Ruptured } \\
(\mathrm{N}=82)\end{array}$ & $\begin{array}{l}\text { Unruptured } \\
(\mathrm{N}=37)\end{array}$ & $\begin{array}{l}\text { Univariate } \\
\text { analysis (P) }\end{array}$ & $\begin{array}{l}\text { Multivariate } \\
\text { analysis }(\mathrm{P})\end{array}$ & $\begin{array}{c}\text { Multivariate } \\
\text { analysis [OR }(95 \% \mathrm{CI})]\end{array}$ \\
\hline Age (years) & $56.74 \pm 11.689$ & $51.46 \pm 11.770$ & 0.028 & 0.066 & $1.034(0.998-1.071)$ \\
\hline Female & $52(63.4)$ & $18(48.6)$ & 0.130 & - & - \\
\hline Hypertension & $58(70.7)$ & $18(48.6)$ & 0.020 & 0.055 & $2.241(0.984-5.104)$ \\
\hline Hypercholesterolemia & $17(20.7)$ & $7(18.9)$ & 0.820 & - & - \\
\hline Diabetes & $13(15.9)$ & $4(10.8)$ & 0.476 & - & - \\
\hline Smoking & $27(32.9)$ & $14(37.8)$ & 0.602 & - & - \\
\hline Alcohol & $21(25.6)$ & $9(24.3)$ & 0.881 & - & - \\
\hline Heart disease & $11(13.4)$ & $2(5.4)$ & 0.195 & - & - \\
\hline
\end{tabular}

OR: odds ratio, CI: confidence interval. 


\section{Results of SNP analyses}

Until recently, the exact etiology of intracranial aneurysm formation was unclear. Most experts now believe that IA is a complex disorder, which is affected by environmental and genetic factors, the former including smoking, hypertension, alcohol consumption, age, gender, etc. To examine the contribution of genetic factors to the pathogenesis of IA, we studied 28 SNPs from 22 genes (Table 4). Using the Pearson $\chi^{2}$ test for 28 SNP loci, we found that IA is associated with two SNPs: in the TSLC2A9 gene, rs7660895 (P = 0.007; OR, 1.541; 95\%CI, 1.126-2.110); and in the TOX gene, rs11777927 ( $\mathrm{P}=0.013$; OR, 1.511; $95 \%$ CI, 1.088-2.098) (Table 5). When subjects had a family relationship, we only recruited one member for the analysis. Following removal of the influence of patient relatedness, this study revealed rs7660895 ( $\mathrm{P}=0.001 ; \mathrm{OR}, 1.691 ; 95 \% \mathrm{CI}, 1.226-2.332)$ and $\mathrm{rs} 11777927(\mathrm{P}=$ 0.006 ; OR, $1.587 ; 95 \% \mathrm{CI}, 1.137-2.213$ ) to be significantly associated with increased risk of IA (Table 6). We also compared the allele frequencies between patients having ruptured and unruptured aneurysms and found that the minor allele of rs 7660895 (A) (P=0.007; OR, 2.196; 95\% CI, 1.230-3.921) was associated with IA rupture. No association was detected between rs11777927 and the rupture of IAs (Table 7).

\begin{tabular}{lcrcr}
\multicolumn{1}{c}{ Table 4. Twenty-eight SNP loci. } & & & \\
\hline Gene & SNP & Chromosome & PAF \\
\hline SLITRK5 & rs371276 & 13 & 89830501 & 0.2443 \\
NUB1 & rs446886 & 7 & 151053043 & 0.2984 \\
FTO & rs708254 & 16 & 54123389 & 0.3898 \\
ESR1 & rs722208 & 6 & 152322885 & 0.4123 \\
MYH9 & rs739097 & 22 & 36746079 & 0.4949 \\
SLC2A9 & rs1014290 & 4 & 10001861 & 0.3076 \\
SLC17A1 & rs1165196 & 6 & 25813150 & 0.2599 \\
TOX & rs1526167 & 8 & 59702355 & 0.4775 \\
intergenic & rs1581498 & 7 & 22908243 & 0.3783 \\
FTO & rs1876942 & 16 & 54118435 & 0.4789 \\
ABCG2 & rs2231142 & 4 & 89052323 & 0.1391 \\
MYH9 & rs2269532 & 22 & 36718039 & 0.3903 \\
PDGFB & rs2285094 & 22 & 39622560 & 0.3283 \\
SFI1 & rs2295251 & 3 & 32013265 & 0.4040 \\
PCAF & rs2929402 & 21 & 20096110 & 0.4187 \\
KCNJ15 & rs3746876 & 10 & 39671447 & 0.0459 \\
SIRT1 & rs3758391 & 12 & 69643342 & 0.4729 \\
IGF1 & rs7312112 & 1 & 103046823 & 0.4624 \\
CAMTA1 & rs7546903 & 4 & 6936272 & 0.3678 \\
SLC2A9 & rs7660895 & 10 & 9985445 & 0.3517 \\
TCF7L2 & rs7903146 & 6 & 114758349 & 0.2902 \\
PKHD1 & rs9395706 & 9 & 51544360 & 0.2957 \\
CDKN2A/B & rs10811661 & 22134094 & 0.2062 \\
TBX5 & rs11067076 & 8 & 114799863 & 0.1928 \\
TOX & rs11777927 & 8 & 59881039 & 0.2563 \\
SLC30A8 & rs13266634 & 118184783 & 0.2824 \\
TOX & rs17304270 & 59979034 & 0.3903 \\
ABI2 & rs62183937 & 204193688 & 0.2580 \\
\hline & & &
\end{tabular}


Table 5. SNP genotypes and allele frequencies in patients and controls.

\begin{tabular}{|c|c|c|c|c|c|c|c|c|c|}
\hline SNP & Genotype & $\begin{array}{l}\text { Patients with } \\
\text { IA [N }(\%)]\end{array}$ & $\begin{array}{l}\text { Controls } \\
{[N(\%)]}\end{array}$ & Allele & IA [N (\%)] & $\begin{array}{l}\text { Controls } \\
\mathrm{N}(\%)\end{array}$ & $\mathrm{P}$ & OR & $95 \% \mathrm{CI}$ \\
\hline \multirow[t]{3}{*}{ rs371276 } & $\mathrm{CC}$ & $36(31.0)$ & $82(32.7)$ & $\mathrm{C}$ & $132(56.9)$ & $295(58.8)$ & 0.633 & 0.926 & $0.676-1.269$ \\
\hline & $\mathrm{TC}$ & $60(51.7)$ & $131(52.2)$ & $\mathrm{T}$ & $100(43.1)$ & $207(41.2)$ & & & \\
\hline & TT & $20(17.2)$ & $38(15.1)$ & & & & & & \\
\hline \multirow[t]{3}{*}{ rs446886 } & AA & $3(2.6)$ & $2(0.8)$ & $\mathrm{T}$ & $25(10.9)$ & $39(7.8)$ & 0.178 & 1.435 & $0.846-2.435$ \\
\hline & $\mathrm{AG}$ & $19(16.5)$ & $35(14.1)$ & G & $205(89.1)$ & $459(92.2)$ & & & \\
\hline & GG & $93(80.9)$ & $212(85.1)$ & & & & & & \\
\hline \multirow[t]{3}{*}{ rs708254 } & $\mathrm{AA}$ & $19(16.4)$ & $34(13.5)$ & G & $92(39.7)$ & 194 (38.6) & 0.794 & 1.043 & $0.759-1.435$ \\
\hline & GA & $54(46.6)$ & $126(50.2)$ & A & $140(60.3)$ & $308(61.4)$ & & & \\
\hline & GG & $43(37.1)$ & $91(36.3)$ & & & & & & \\
\hline \multirow{3}{*}{ rs722208 } & $\mathrm{AA}$ & $23(19.8)$ & $51(20.3)$ & G & $101(43.5)$ & $217(43.2)$ & 0.938 & 1.013 & $0.740-1.386$ \\
\hline & GA & $55(47.4)$ & $115(45.8)$ & A & $131(56.5)$ & $285(56.8)$ & & & \\
\hline & GG & $38(32.8)$ & 85 (33.9) & & & & & & \\
\hline \multirow[t]{3}{*}{ rs739097 } & AA & $2(1.8)$ & $16(6.4)$ & A & $41(18.1)$ & $115(23.0)$ & 0.140 & 0.742 & $0.499-1.104$ \\
\hline & GA & $37(32.7)$ & $83(33.2)$ & G & $185(81.9)$ & $385(77.0)$ & & & \\
\hline & GG & $74(65.5)$ & $151(60.4)$ & & & & & & \\
\hline \multirow[t]{3}{*}{ rs 1014290} & $\mathrm{CC}$ & $20(17.2)$ & $38(15.1)$ & $\mathrm{C}$ & $97(41.8)$ & $189(37.6)$ & 0.282 & 1.190 & $0.866-1.634$ \\
\hline & $\mathrm{TC}$ & $57(49.1)$ & $113(45.0)$ & $\mathrm{T}$ & $135(58.2)$ & $313(62.4)$ & & & \\
\hline & TT & $39(33.6)$ & $100(39.8)$ & & & & & & \\
\hline \multirow[t]{3}{*}{ rs1165196 } & $\mathrm{CC}$ & $4(3.6)$ & $15(6.0)$ & $\mathrm{C}$ & $41(18.3)$ & $101(20.3)$ & 0.536 & 0.881 & $0.589-1.318$ \\
\hline & $\mathrm{CT}$ & $33(29.5)$ & $71(28.8)$ & $\mathrm{T}$ & $183(81.7)$ & $397(7.7)$ & & & \\
\hline & TT & $75(67.0)$ & $163(65.9)$ & & & & & & \\
\hline \multirow[t]{3}{*}{ rs 1526167} & AA & $30(26.5)$ & $70(27.9)$ & A & $117(51.8)$ & $257(51.2)$ & 0.886 & 1.023 & $0.747-1.401$ \\
\hline & GA & $57(50.4)$ & $117(46.6)$ & G & $109(48.2)$ & $245(48.8)$ & & & \\
\hline & GG & $26(23.0)$ & $64(25.5)$ & & & & & & \\
\hline \multirow[t]{3}{*}{ rs 1581498} & $\mathrm{CC}$ & $20(17.9)$ & 29 (11.6) & $\mathrm{C}$ & $87(38.8)$ & $181(36.1)$ & 0.473 & 1.126 & $0.814-1.558$ \\
\hline & $\mathrm{TC}$ & $47(42.0)$ & $123(49.0)$ & $\mathrm{T}$ & $137(61.2)$ & $321(63.9)$ & & & \\
\hline & TT & $45(40.2)$ & $99(39.4)$ & & & & & & \\
\hline \multirow[t]{3}{*}{ rs 1876942} & GG & $41(35.7)$ & $86(34.4)$ & $\mathrm{G}$ & 137 (59.6) & $300(60.0)$ & 0.911 & 0.982 & $0.714-1.350$ \\
\hline & GT & $55(47.8)$ & $128(51.2)$ & $\mathrm{T}$ & $93(40.4)$ & $200(40.0)$ & & & \\
\hline & TT & $19(16.5)$ & $36(14.4)$ & & & & & & \\
\hline \multirow[t]{3}{*}{ rs 2231142} & AA & $10(8.6)$ & $23(9.2)$ & A & $82(35.3)$ & $158(31.5)$ & 0.299 & 1.190 & $0.857-1.653$ \\
\hline & $\mathrm{CA}$ & $62(53.4)$ & $112(44.6)$ & $\mathrm{C}$ & $150(64.7)$ & $344(68.5)$ & & & \\
\hline & $\mathrm{CC}$ & $44(37.9)$ & $116(46.2)$ & & & & & & \\
\hline \multirow{3}{*}{ rs 2269532} & AA & $70(60.3)$ & $145(57.8)$ & $\mathrm{A}$ & $184(79.3)$ & $381(75.9)$ & 0.307 & 1.217 & $0.834-1.776$ \\
\hline & $\mathrm{AG}$ & $44(37.9)$ & $91(36.3)$ & $\mathrm{G}$ & $48(20.7)$ & $21(24.1)$ & & & \\
\hline & GG & $2(1.7)$ & $15(6.0)$ & & & & & & \\
\hline \multirow[t]{3}{*}{ rs2285094 } & AA & $83(71.6)$ & $159(63.3)$ & A & $196(84.5)$ & $399(79.5)$ & 0.108 & 1.405 & $0.927-2.131$ \\
\hline & GA & $30(25.9)$ & $81(32.3)$ & G & $36(15.5)$ & $103(20.5)$ & & & \\
\hline & GG & $3(2.6)$ & $11(4.4)$ & & & & & & \\
\hline rs2295251 & AA & $35(30.2)$ & $97(38.6)$ & A & $133(57.3)$ & $325(64.7)$ & 0.054 & 0.732 & $0.532-1.006$ \\
\hline & $\mathrm{AG}$ & $63(54.3)$ & $131(52.2)$ & G & $99(42.7)$ & $177(35.3)$ & & & \\
\hline & GG & $18(15.5)$ & $23(9.2)$ & & & & & & \\
\hline rs2929402 & AA & $21(18.1)$ & $32(12.7)$ & A & $98(42.2)$ & $193(38.4)$ & 0.328 & 1.171 & $0.853-1.607$ \\
\hline & GA & $56(48.3)$ & $129(51.4)$ & G & $134(57.8)$ & 309 (61.6) & & & \\
\hline & GG & $39(33.6)$ & $90(35.9)$ & & & & & & \\
\hline rs3746876 & $\mathrm{CC}$ & $98(84.5)$ & $204(81.3)$ & $\mathrm{C}$ & $212(91.4)$ & $453(90.2)$ & 0.623 & 1.147 & $0.665-1.977$ \\
\hline & $\mathrm{CT}$ & $16(13.8)$ & $45(17.9)$ & $\mathrm{T}$ & $20(8.6)$ & $49(9.8)$ & & & \\
\hline & TT & $2(1.7)$ & $2(0.8)$ & & & & & & \\
\hline rs3758391 & $\mathrm{CC}$ & $3(2.6)$ & $8(3.2)$ & $\mathrm{C}$ & $38(16.4)$ & 97 (19.3) & 0.339 & 0.818 & $0.542-1.235$ \\
\hline & $\mathrm{TC}$ & $32(27.6)$ & $81(32.3)$ & $\mathrm{T}$ & 194 (83.6) & $405(80.7)$ & & & \\
\hline & TT & $81(69.8)$ & $162(64.5)$ & & & & & & \\
\hline rs7312112 & GG & $41(36.3)$ & 75 (29.9) & G & $126(55.8)$ & $270(53.8)$ & 0.622 & 1.083 & $0.790-1.485$ \\
\hline & GT & $44(38.9)$ & $120(47.8)$ & $\mathrm{T}$ & $100(44.2)$ & $232(46.2)$ & & & \\
\hline & TT & $28(24.8)$ & $56(22.3)$ & & & & & & \\
\hline rs7546903 & AA & $16(13.8)$ & $36(14.3)$ & A & $87(37.5)$ & $186(37.1)$ & 0.907 & 1.019 & $0.739-1.406$ \\
\hline & GA & $55(47.4)$ & $114(45.4)$ & $\mathrm{G}$ & $145(62.5)$ & $316(62.9)$ & & & \\
\hline & GG & $45(38.8)$ & $101(40.2)$ & & & & & & \\
\hline rs7660895 & $\mathrm{AA}$ & $53(45.7)$ & $84(33.5)$ & A & $134(57.8)$ & $236(47.0)$ & 0.007 & 1.541 & $1.126-2.110$ \\
\hline & $\mathrm{AG}$ & $28(24.1)$ & $68(27.1)$ & G & $98(42.2)$ & $266(53.0)$ & & & \\
\hline & GG & $35(30.2)$ & $99(39.4)$ & & & & & & \\
\hline rs7903146 & $\mathrm{CC}$ & $100(90.9)$ & $222(88.4)$ & $\mathrm{C}$ & $210(95.5)$ & $473(94.2)$ & 0.500 & 1.288 & $0.616-2.690$ \\
\hline
\end{tabular}

Continued on next page 


\begin{tabular}{|c|c|c|c|c|c|c|c|c|c|}
\hline SNP & Genotype & $\begin{array}{l}\text { Patients with } \\
\text { IA [N (\%)] }\end{array}$ & $\begin{array}{l}\text { Controls } \\
{[\mathrm{N}(\%)]}\end{array}$ & Allele & IA [N (\%)] & $\begin{array}{l}\text { Controls } \\
{[\mathrm{N}(\%)]}\end{array}$ & $P$ & OR & $95 \% \mathrm{CI}$ \\
\hline & $\mathrm{TC}$ & $10(9.1)$ & $29(11.6)$ & $\mathrm{T}$ & $10(4.5)$ & $29(5.8)$ & & & \\
\hline \multirow[t]{3}{*}{ rs9395706 } & GG & $21(19.3)$ & $27(10.8)$ & G & $91(41.7)$ & $177(35.3)$ & 0.098 & 1.316 & $0.950-1.822$ \\
\hline & GT & $49(45.0)$ & $123(49.0)$ & $\mathrm{T}$ & $127(58.3)$ & $325(64.7)$ & & & \\
\hline & TT & $39(35.8)$ & $101(40.2)$ & & & & & & \\
\hline \multirow[t]{3}{*}{ rs10811661 } & $\mathrm{CC}$ & $23(19.8)$ & $74(29.5)$ & $\mathrm{C}$ & $108(46.6)$ & $268(53.4)$ & 0.085 & 0.760 & $0.557-1.039$ \\
\hline & $\mathrm{TC}$ & $62(53.4)$ & $120(47.8)$ & $\mathrm{T}$ & $124(53.4)$ & $234(46.6)$ & & & \\
\hline & TT & $31(26.7)$ & $57(22.7)$ & & & & & & \\
\hline \multirow[t]{2}{*}{ rs11067076 } & AA & $109(94.0)$ & 234 (93.2) & A & $225(97.0)$ & 485 (96.6) & 0.794 & 1.127 & $0.461-2.755$ \\
\hline & TA & $7(6.0)$ & $17(6.8)$ & $\mathrm{T}$ & $7(3.0)$ & $17(3.4)$ & & & \\
\hline \multirow{3}{*}{ rs11777927 } & AA & $14(12.1)$ & $21(8.4)$ & A & $88(37.9)$ & $144(28.8)$ & 0.013 & 1.511 & $1.088-2.098$ \\
\hline & AT & $60(51.7)$ & $102(40.8)$ & $\mathrm{T}$ & $144(62.1)$ & $356(71.2)$ & & & \\
\hline & TT & $42(36.2)$ & $127(50.8)$ & & & & & & \\
\hline \multirow[t]{3}{*}{ rs13266634 } & $\mathrm{CC}$ & $38(32.8)$ & $79(31.7)$ & $\mathrm{C}$ & $138(59.5)$ & $286(57.4)$ & 0.601 & 1.088 & $0.793-1.494$ \\
\hline & $\mathrm{CT}$ & $62(53.4)$ & $128(51.4)$ & $\mathrm{T}$ & $94(40.5)$ & $212(42.6)$ & & & \\
\hline & $\mathrm{TT}$ & $16(13.8)$ & $42(16.9)$ & & & & & & \\
\hline \multirow[t]{3}{*}{ rs 17304270} & AA & $92(86.8)$ & $214(85.3)$ & A & $197(92.9)$ & $464(92.4)$ & 0.818 & 1.076 & $0.578-2.000$ \\
\hline & $\mathrm{AG}$ & $13(12.3)$ & $36(14.3)$ & G & $15(7.1)$ & $38(7.6)$ & & & \\
\hline & GG & $1(0.9)$ & $1(0.4)$ & & & & & & \\
\hline \multirow[t]{3}{*}{ rs62183927 } & GG & $39(33.6)$ & $78(31.1)$ & G & $141(60.8)$ & $284(56.6)$ & 0.284 & 1.189 & $0.866-1.633$ \\
\hline & GT & $63(54.3)$ & $128(51.0)$ & $\mathrm{T}$ & $91(39.2)$ & $218(43.4)$ & & & \\
\hline & $\mathrm{TT}$ & $14(12.1)$ & $45(17.9)$ & & & & & & \\
\hline
\end{tabular}

Table 6. Allele frequencies after removal of data from related patients.

\begin{tabular}{|c|c|c|c|c|c|c|}
\hline SNP & Allele & Patients with IA [N (\%)] & Controls [N (\%)] & $\mathrm{P}$ & OR & $95 \% \mathrm{CI}$ \\
\hline \multirow[t]{2}{*}{ rs7660895 } & A & $132(60.0)$ & $236(47.0)$ & 0.001 & 1.691 & $1.226-2.332$ \\
\hline & G & $88(40.0)$ & $266(53.0)$ & & & \\
\hline \multirow[t]{2}{*}{ rs11777927 } & A & $86(39.1)$ & $144(28.8)$ & 0.006 & 1.587 & $1.137-2.213$ \\
\hline & $\mathrm{T}$ & $134(60.9)$ & $356(71.2)$ & & & \\
\hline
\end{tabular}

Table 7. SNP genotype and allele frequencies in ruptured and unruptured aneurysms.

\begin{tabular}{|c|c|c|c|c|c|c|c|c|c|}
\hline SNP & Genotype & $\begin{array}{c}\text { Ruptured } \\
{[\mathrm{N}(\%)]}\end{array}$ & $\begin{array}{c}\text { Unruptured } \\
{[\mathrm{N}(\%)]}\end{array}$ & Allele & $\begin{array}{c}\text { Ruptured } \\
{[\mathrm{N}(\%)]}\end{array}$ & $\begin{array}{c}\text { Unruptured } \\
{[\mathrm{N}(\%)]}\end{array}$ & $\mathrm{P}$ & OR & $95 \% \mathrm{CI}$ \\
\hline \multirow[t]{3}{*}{ rs7660895 } & AA & $42(50.6)$ & $11(33.3)$ & A & $105(63.3)$ & $29(43.9)$ & 0.007 & 2.196 & $1.230-3.921$ \\
\hline & $\mathrm{AG}$ & $21(25.3)$ & $7(21.2)$ & G & $61(36.7)$ & $37(56.1)$ & & & \\
\hline & GG & $20(24.1)$ & $15(45.5)$ & & & & & & \\
\hline \multirow[t]{3}{*}{ rs11777927 } & AA & $11(13.4)$ & $3(8.8)$ & A & $64(39.0)$ & $24(35.3)$ & 0.594 & 1.173 & $0.652-2.113$ \\
\hline & AT & $42(51.2)$ & $18(52.9)$ & $\mathrm{T}$ & 100 (61.0) & $44(64.7)$ & & & \\
\hline & TT & $29(35.4)$ & $13(38.2)$ & & & & & & \\
\hline
\end{tabular}

\section{DISCUSSION}

IAs are common lesions in the brain, a fraction of which rupture and lead to SAH with devastating consequences. In a population consisting of $50 \%$ men, with a mean age of 50 years and without comorbidity, the prevalence of unruptured IAs (UIAs) is $3.2 \%$. The prevalence of UIAs is higher in patients with autosomal dominant polycystic kidney disease (ADPKD) or a positive family history of intracranial SAH than in people without comorbidity. Women have a higher prevalence of UIAs than men, which can mainly be attributed to the relatively more number of women older than 50 years (Vlak et al., 2011). Until recently, the exact etiology of intracranial aneurysm formation has remained unclear. 
A meta-analysis of all longitudinal and case-control studies for identifying risk factors for SAH from 1966 to 2005 concluded that environmental risk factors included smoking, excessive alcohol consumption, and hypertension (Feigin et al., 2005). Inflammatory and immunological reactions were also thought to be related to IA formation and rupture. In addition to ambient factors, epidemiological studies have demonstrated a familial influence contributing toward the pathogenesis of IAs, with an increased frequency in first- and second-degree relatives of people with SAH (Kissela et al., 2002).

In our study, hypertension significantly increased the risk of IA formation, whereas the effects of cigarette smoking, heart disease, hypercholesterolemia, T2DM, and daily drinking were insignificant. Other studies have also found that hypertension was a major risk factor for IA (Rasing et al., 2012; Vlak et al., 2013a). However, our data on smoking contradict previous findings. Many prior studies have found that smoking independently increased the risk of IAs (Vlak et al., 2013a), and smoking and a history of hypertension were the strongest independent risk factors for IA. Furthermore, these studies found that the joint risk of smoking and hypertension was higher than the sum of the individual risks for either factor (Vlak et al., 2013a).

By univariate analysis, we found that hypertension and age were insignificant factors in the rupture of IAs. Previous studies have reported that smoking, migraine, and hypercholesterolemia act as independent risk factors for aneurismal rupture. However, although a history of hypertension does not increase risk of rupture, a sudden rise in blood pressure might still trigger aneurismal rupture (Vlak et al., 2013b). Age, gender, size of the unruptured IA, site, and type of aneurysm were all considered important factors in determining the risk for subsequent aneurysm rupture (Wermer et al., 2007). In addition, a history of SAH and posterior circulation aneurysms were also found to be significant risk factors for the prediction of intracranial saccular aneurysm rupture (Ishibashi et al., 2009).

The objective of this study was to investigate the genetic markers related to genes for T2DM (CAMTA1, SLC30A8, CDKN2A/B, TCF7L2, SLC2A9, IGF1, ESR1, PDGFB, KCNJ15, and $S F I 1)$, hyperlipidemia (CDKN2A/B,ABI2, and SIRT1), gout (SLC17A1 and ABCG2), obesity (FTO), inflammation (PCAF), hypertension (MYH9, ESR1, and PDGFB), ADPKD (PKHD1), and growth and metabolism (TBX5, NUB1, and SLITRK5) for their contribution to the risks of IA.

The functions of these genes and their products, as well as their association with IA risk factors, are as follows: CAMTA1, the calmodulin binding transcription activator 1, was shown by the French prospective D.E.S.I.R. study, a recently published GWA analysis, to have a nominal to strong association with T2DM (Cauchi et al., 2008). SLC30A8, encoding the islet zinc transporter ZnT8, is necessary for zinc flux into $\beta$-cell insulin-secretory granules and subsequent insulin crystallization. A previously identified common SLC30A8 missense variant is associated with T2DM risk at significance levels beyond genome-wide thresholds (Scott et al., 2007; Sladek et al., 2007; Cauchi et al., 2008). CDKN2A/B encodes Pl6 ${ }^{\mathrm{INK} 4 \mathrm{a}}$ and P15 ${ }^{\text {INK4a }}$, which function as inhibitors of CDK4 and CDK5 kinase, respectively. $C D K N 2 A / B$ has not only been associated with T2DM (Scott et al., 2007; Cauchi et al., 2008), but also with atherosclerosis (Motterle et al., 2012). TCF7L2, the transcription factor 7-like 2 gene, has been associated with T2DM, and its variants have been shown to confer T2DM risk (Scott et al., 2007; Sladek et al., 2007). SLC2A9 encodes a facilitative glucose transporter. Phenotypic switching of vascular smooth muscle cells (VSMCs) from the quiescent, contractile state to the active, synthetic state is facilitated by systemic and locally released trophic factors at the site of vascular lesion (Owens et al., 2004). The initiating event toward increased glucose metabolism in proliferative VSMCs has been shown to be the cellular uptake of glucose via facili- 
tative glucose transporters (Suzuki et al., 2001). The insulin-like growth factor IGF1 is known to alter the phenotype of smooth muscle cells, and is a strong mitogen and chemo-attractant for these cells. Excess of IGF-1 can affect the cerebral vascular wall, resulting in aneurysm formation (Oshino et al., 2013). Polymorphisms in ESR1, encoding an estrogen receptor, may affect the onset age for stroke and the probability of developing cerebrovascular disease (Lazaros et al., 2008). Signaling through the estrogen receptor alpha regulates vasodilatation and atherogenesis. As hypertension and atherosclerosis are major mechanisms underlying stroke development, genetic variants of ESRI may be associated with intracerebral hemorrhage (Strand et al., 2007; Lazaros et al., 2008). PDGFRB, the platelet-derived growth factor receptor beta polypeptide gene, encodes a cell surface tyrosine kinase receptor for members of the plateletderived growth factor family, which might play an important role in the pathogenesis of both hypertension and T2DM (Stoynev et al., 2014). The KCNJ15 gene, encoding a potassium channel, was associated with obese T2DM, and genetic variants of $K C N J 15$ have been shown to reduce insulin secretion (Fukuda et al., 2013). The SFI1 gene, encoding a homolog of the yeast suppressor of the cAMP pathway/spindle assembly protein, has been associated with diabetic nephropathy (McDonough et al., 2011).

The $A B I 2$ gene encodes a tumor suppressor and cell migration inhibitor. It has been implicated in vascular disease through a near-significant association with arterial intima-medial thickness (O'Donnell et al., 2007). SIRT1 (a NAD+ dependent deacetylase) and its activators have been put forward as novel therapeutic targets for age-related vascular diseases (Wang et al., 2013). The SLC17A1 protein (NPT1) was the first identified member of the SLC17 phosphate transporter family, shown to be responsible for renal urate excretion (Dalbeth et al., 2014). $A B C G 2$, encoding a uric acid transporter, was identified to be associated with uric acid concentration and gout (Dehghan et al., 2008); its variants reduce the excretion of uric acid by the kidney. However, the genetic urate score analysis did not provide evidence for an association between serum urate and cardiovascular risk factors or coronary heart disease (Yang et al., 2010). FTO, encoding a nuclear protein of the AlkB-related non-heme iron and 2-oxoglutarate-dependent oxygenase superfamily, was identified as an obesity-related gene, and which increased obesity-related vascular diseases (Al-Attar et al., 2008). PCAF has histone acetylating activity, promotes transcription of multiple inflammatory genes, and acts as master switch that stimulates multiple inflammatory processes. Its deficiency reportedly results in a repressed in vitro inflammatory response in many cell types known to be involved in arteriogenesis (Bastiaansen et al., 2013). MYH9 is associated with a spectrum of kidney diseases in African Americans, including essential hypertension and nephropathy attributed to hypertension, and focal segmental glomerulosclerosis (Murea and Freedman, 2010). Genes with broad or general functions include $T B X 5$, a member of the T-box transcription factor family; NUB1, a ubiquitin-like protein shown to conjugate with cullin family members in order to regulate vital biological events; and SLITRK5, encoding an integral membrane protein. Finally, PKHD1 has been shown to be associated with ADPKD (Bergmann et al., 2005).

Although the roles of several genes were associated with the vessel wall directly, or related to risk factors, we found that IA was associated with only two SNPs; in the TSL$C 2 A 9$ gene: rs7660895 ( $\mathrm{P}=0.007$; OR, 1.541; 95\%CI, 1.126-2.110), and in the TOX gene: rs11777927 ( $\mathrm{P}=0.013$; OR, 1.511; 95\%CI, 1.088-2.098). Following removal of the confounding factor of familial relatedness, this study revealed that $\mathrm{rs} 7660895(\mathrm{P}=0.001$; OR, $1.691 ; 95 \% \mathrm{CI}, 1.226-2.332)$ and $\mathrm{rs} 11777927$ ( $\mathrm{P}=0.006$; OR, 1.587; 95\%CI, 1.137-2.213) were significantly associated with increased risk of IA (Table 6), and also found that the mi- 
nor allele of rs7660895(A) was associated with rupture of IA as well ( $\mathrm{P}=0.007$; OR, 2.196; 95\%CI, 1.230-3.921) (Table 7).

The TOX gene is located on human chromosome 8q12. TOX encodes a member of the class of high mobility group box proteins, which can bind to DNA and thus participate in the regulation of gene expression, such as in transcription, replication, and DNA repair. TOX may play a role in the regulation of expression of certain genes involved in cell cycle progression, such as the cell division cycle gene CDC family and oncogenes (Eymin and Gazzeri, 2010), thereby regulating cell proliferation. TOX expression levels are elevated in thymus cells and leukemia cell lines; meanwhile, TOX has been shown to influence the process of T cell development and differentiation (Wilkinson et al., 2002), and also may be associated with the production of B lymphocytes. At the same time, it has been found to be associated with diabetes. It is possible that inflammatory responses mediated by immune cells, developmentally regulated by $T O X$, contribute to the formation of IAs.

SLC2A9 encodes a facilitative glucose transporter. Phenotypic switching of VSMCs to the synthetic state is facilitated by systemic and locally released trophic factors at the site of vascular lesion (Owens et al., 2004). As a sign of vascular wall remodeling, vascular smooth muscle proliferation causes a change of compliance. Importantly, growth factor-induced VSMC proliferation is dependent on multiple intracellular signaling events and metabolic pathways, including increased glucose consumption via glycolysis (Suzuki et al., 2001). The increased use of sugar in smooth muscle cells originates from the upregulation of sugar transporters on cell membranes. In turn, diabetes is one of the most important risk factors for cerebrovascular disease (Groves et al., 2006). Macro- and microvascular diabetic complications are primarily due to prolonged exposure to hyperglycemia. The initial trigger, whereby high glucose concentrations alter vascular function, is the imbalance between nitric oxide bioavailability and the accumulation of reactive oxygen species, leading to endothelial dysfunction (Paneni et al., 2013). The dysfunction of endothelial cells (ECs) and smooth muscle cells (SMCs) is the main characteristic of diabetic vascular complications. Histological analyses of human IA has shown a decreased number of ECs and SMCs in the IA wall that results, at least partially, from apoptosis (Frosen et al., 2004). The extracellular matrix (ECM) and vessel wall cells are complementary to each other; therefore, loss of the cells within the vessel wall could lead to decreased ECM production; or, ECM degradation might lead to cellular death. The change of ECs, SMCs, and ECM may be the basis of IA formation. SLC2A9 has been related to diabetes: the rs 7660895 locus has been shown to be associated with aneurysm formation and rupture, a possible reason being that it influences glucose transport at the cell membrane.

FTO was identified through its relationship to obesity, which increased risk of obesityrelated vascular diseases (Al-Attar et al., 2008). Individuals with an FTO polymorphism were shown to have a trend towards increased intima-medial thickness of the carotid artery, which is a clinical marker of atherosclerosis (Kivimaki et al., 2008). FTO, however, was not associated with IA in our study.

$P C A F$ has histone acetylase activity and promotes the transcription of multiple inflammatory genes (Bastiaansen et al., 2013). One of the crucial players in the pathophysiology of IA is inflammation. ECs will become activated after injury, subsequently triggering the recruitment of immune cells and production of inflammatory mediators and cytokines, which have been shown to be involved in changes in the smooth muscle membrane and extracellular matrix (O'Neill and Hardie, 2013). In rats, progression of the inflammatory zone and IA formation progressed in parallel (Jamous et al., 2007). Immunohistochemical analyses of human 
IA revealed macrophage and lymphocyte infiltration, with increased expression of inflammatory cytokines; however, $P C A F$ was not associated with IA in our study.

$K C N J 15$ and $T C F 7 L 2$ have been shown to have a significant association with diabetes (Fukuda et al., 2013; Sladek et al., 2007). $C D K N 2 A / B$ has not only been demonstrated to be associated with T2DM (Cauchi et al., 2008; Scott et al., 2007), but also with atherosclerosis (Motterle et al., 2012). None of these, however, was associated with IA in our study. The correlation between diabetes and the mechanism of IA formation, development, and rupture still needs further examination and discussion in future studies.

Despite the previously described difficulties in identifying gene variants associated with IA, knowledge of the genetic determinants for IAs may provide insight into the development of aneurysms, clues toward stopping aneurysm formation, and diagnostic tools for identifying individuals at increased risk. Further multicenter studies have to be carried out in order to narrow down the responsible genetic factors, which will lead to a better understanding of the mechanism of IA formation. Finally, future studies should also seek to detect genetic determinants of aneurysm rupture in order to aid in the selection of patients for preventive treatment, and improve the substantial morbidity and mortality currently associated with IAs.

\section{Conflicts of interest}

The authors declare no conflict of interest.

\section{ACKNOWLEDGMENTS}

We thank Weidong Li, Fengjiang Wei, and Zhen Zhang for their help with the statistical analyses. We would like to thank the Department of Neurosurgery, Tianjin Medical University General Hospital.

\section{REFERENCES}

Al-Attar SA, Pollex RL, Ban MR, Young TK, et al. (2008). Association between the FTO rs9939609 polymorphism and the metabolic syndrome in a non-Caucasian multi-ethnic sample. Cardiovasc. Diabetol. 7: 5.

Bastiaansen A J, Ewing MM, de Boer HC, van der Pouw Kraan T C, et al. (2013). Lysine acetyltransferase PCAF is a key regulator of arteriogenesis. Arterioscler. Thromb. Vasc. Bio. 33: 1902-1910.

Bergmann C, Kupper F, Schmitt CP, Vester U, et al. (2005). Multi-exon deletions of the PKHD1 gene cause autosomal recessive polycystic kidney disease (ARPKD). J. Med. Genet. 42: e63.

Cauchi S, Proença C, Choquet H, Gaget S, et al. (2008). Analysis of novel risk loci for type 2 diabetes in a general French population: the D.E.S.I.R. study. J. Mol. Med. 86: 341-348.

Dalbeth N, House ME, Gamble GD, Horne A, et al. (2014). Population-specific effects of SLC17A1 genotype on serum urate concentrations and renal excretion of uric acid during a fructose load. Ann. Rheum. Dis. 73: 313-314.

Dehghan A, Kottgen A, Yang Q, Hwang SJ, et al. (2008). Association of three genetic loci with uric acid concentration and risk of gout: a genome-wide association study. Lancet 372: 1953-1961.

Eymin B and Gazzeri S (2010). Role of cell cycle regulators in lung carcinogenesis. Cell Adh. Migr. 4: 114-123.

Feigin VL, Rinkel GJ, Lawes CM, Algra A, et al. (2005). Risk factors for subarachnoid hemorrhage: an updated systematic review of epidemiological studies. Stroke 36: 2773-2780.

Frosen J, Piippo A, Paetau A, Kangasniemi M, et al. (2004). Remodeling of saccular cerebral artery aneurysm wall is associated with rupture: histological analysis of 24 unruptured and 42 ruptured cases. Stroke 35: 2287-2293.

Fukuda H, Imamura M, Tanaka Y, Iwata M, et al. (2013). Replication study for the association of a single-nucleotide polymorphism, rs3746876, within KCNJ15, with susceptibility to type 2 diabetes in a Japanese population. J. Hum. Genet. 58: 490-493. 
Groves CJ, Zeggini E, Minton J, Frayling TM, et al. (2006). Association analysis of 6,736 U.K. subjects provides replication and confirms TCF7L2 as a type 2 diabetes susceptibility gene with a substantial effect on individual risk. Diabetes 55: 2640-2644.

Ishibashi T, Murayama Y, Urashima M, Saguchi T, et al. (2009). Unruptured intracranial aneurysms: incidence of rupture and risk factors. Stroke 40: 313-316.

Jamous MA, Nagahiro S, Kitazato KT, Tamura T, et al. (2007). Endothelial injury and inflammatory response induced by hemodynamic changes preceding intracranial aneurysm formation: experimental study in rats. J. Neurosurg. 107: 405-411.

Katati MJ, Santiago-Ramajo S, Perez-Garcia M, Meersmans-Sanchez Jofre M, et al. (2007). Description of quality of life and its predictors in patients with aneurysmal subarachnoid hemorrhage. Cerebrovasc. Dis. 24: 66-73.

Kissela BM, Sauerbeck L, Woo D, Khoury J, et al. (2002). Subarachnoid hemorrhage: a preventable disease with a heritable component. Stroke 33: 1321-1326.

Kivimaki M, Smith GD, Timpson NJ, Lawlor DA, et al. (2008). Lifetime body mass index and later atherosclerosis risk in young adults: examining causal links using Mendelian randomization in the Cardiovascular Risk in Young Finns study. Eur. Heart. J. 29: 2552-2560.

Lazaros L, Markoula S, Xita N, Giannopoulos S, et al. (2008). Association of estrogen receptor-alpha gene polymorphisms with stroke risk in patients with metabolic syndrome. Acta. Neurol. Scand. 117: 186-190.

McDonough CW, Palmer ND, Hicks PJ, Roh BH, et al. (2011). A genome-wide association study for diabetic nephropathy genes in African Americans. Kidney Int. 79: 563-572.

Motterle A, Pu X, Wood H, Xiao Q, Gor S, et al. (2012). Functional analyses of coronary artery disease associated variation on chromosome 9p21 in vascular smooth muscle cells. Hum. Mol. Genet. 21: 4021-4029.

Murea M and Freedman BI (2010). Essential hypertension and risk of nephropathy: a reappraisal. Curr. Opin. Nephrol. Hypertens. 19: 235-241.

O’Donnell CJ, Cupples LA, D'Agostino RB, Fox CS, et al. (2007). Genome-wide association study for subclinical atherosclerosis in major arterial territories in the NHLBI's Framingham Heart Study. BMC Med. Genet. (Suppl. 8) 1: S4.

O'Neill LA and Hardie DG (2013). Metabolism of inflammation limited by AMPK and pseudo-starvation. Nature 493: 346-355.

Oshino S, Nishino A, Suzuki T, Arita H, et al. (2013). Prevalence of cerebral aneurysm in patients with acromegaly. Pituitary 16: 195-201.

Owens GK, Kumar MS and Wamhoff BR (2004). Molecular regulation of vascular smooth muscle cell differentiation in development and disease. Physiol. Rev. 84: 767-801.

Paneni F, Beckman JA, Creager MA and Cosentino F (2013). Diabetes and vascular disease: pathophysiology, clinical consequences, and medical therapy: part I. Eur. Heart J. 34: 2436-2443.

Rasing I, Nieuwkamp DJ, Algra A and Rinkel GJ (2012). Additional risk of hypertension and smoking for aneurysms in people with a family history of subarachnoid haemorrhage. J. Neurol. Neurosurg. Psychiatry 83: 541-542.

Ruigrok YM, Rinkel GJ and Wijmenga C (2005). Genetics of intracranial aneurysms. Lancet Neurol. 4: 179-189.

Scott LJ, Mohlke KL, Bonnycastle LL, Willer CJ, et al. (2007). A genome-wide association study of type 2 diabetes in Finns detects multiple susceptibility variants. Science 316: 1341-1345.

Sladek R, Rocheleau G, Rung J, Dina C, et al. (2007). A genome-wide association study identifies novel risk loci for type 2 diabetes. Nature 445: 881-885.

Stoynev N, Dimova I, Rukova B, Hadjidekova S, et al. (2014). Gene expression in peripheral blood of patients with hypertension and patients with type 2 diabetes. J. Cardiovasc. Med. 15: 702-709.

Strand M, Soderstrom I, Wiklund PG, Hallmans G, et al. (2007). Estrogen receptor alpha gene polymorphisms and firstever intracerebral hemorrhage. Cerebrovasc. Dis. 24: 500-508.

Suzuki LA, Poot M, Gerrity RG and Bornfeldt KE (2001). Diabetes accelerates smooth muscle accumulation in lesions of atherosclerosis: lack of direct growth-promoting effects of high glucose levels. Diabetes 50: 851-860.

Vlak MH, Algra A, Brandenburg R and Rinkel GJ (2011). Prevalence of unruptured intracranial aneurysms, with emphasis on sex, age, comorbidity, country, and time period: a systematic review and meta-analysis. Lancet. Neurol. 10: 626-636.

Vlak MH, Rinkel GJ, Greebe P and Algra A (2013a). Independent risk factors for intracranial aneurysms and their joint effect: a case-control study. Stroke 44: 984-987.

Vlak MH, Rinkel GJ, Greebe P and Algra A (2013b). Risk of rupture of an intracranial aneurysm based on patient characteristics: a case-control study. Stroke 44: 1256-9.

Wang F, Chen HZ, Lv X and Liu DP (2013). SIRT1 as a novel potential treatment target for vascular aging and age-related vascular diseases. Curr. Mol. Med. 13: 155-64.

Wardlaw JM and White PM (2000). The detection and management of unruptured intracranial aneurysms. Brain 123: 205-21.

Wermer MJ, van der Schaaf IC, Algra A and Rinkel GJ (2007). Risk of rupture of unruptured intracranial aneurysms in relation to patient and aneurysm characteristics: an updated meta-analysis. Stroke 38: 1404-10. 
Wilkinson B, Chen JY, Han P, Rufner KM et al. (2002). TOX: an HMG box protein implicated in the regulation of thymocyte selection. Nat. Immunol. 3: 272-80.

Yang Q, Kottgen A, Dehghan A, Smith AV et al. (2010). Multiple genetic loci influence serum urate levels and their relationship with gout and cardiovascular disease risk factors. Circ. Cardiovasc. Genet. 3: 523-30. 